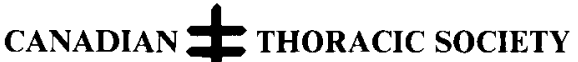 \\ SOCIÉTẼ
}

\section{CLINICAL TRIALS COMMITTEE}

The Clinical Trials Committee is an ad hoc committee of the Canadian Lung Association's Canadian Thoracic Society (CTS) and representation from the Canadian Nursing Respiratory Society, the Canadian Physiotherapy Cardio-respiratory Society and the Respiratory Therapy Society of the Canadian Lung Association. The committee aims to foster national collaborative multicentre research about clinically important questions arising in adult pulmonary medicine; particularly, elaborate studies needed to increase the level of evidence or key recommendations of treatment guidelines produced by the CTS. Other areas of pulmonary medicine, such as pediatrics and thoracic surgery, will be investigated once the committee and its activities are established.

The Clinical Trials Group welcomes letters of intent from the respiratory community for therapeutic, cohort and diagnostic studies. The group currently has representatives from all Canadian universities but also welcomes proposals from community-based physicians. The consortium would especially welcome proposals related to less common respiratory conditions, and wants to facilitate a process to improve the management of these conditions. The terms of reference for the group can be accessed at www.lung.ca/cts, as can a listing of the current institutional representatives and their alternate representatives. In addition, the template for submitting protocols to the committee is located on this Web site.

\section{MEMBERSHIP COMMITTEE}

Some of you may have noted that you have not yet received your membership renewal forms for the $2002-2003$ year. We have experienced some delays in the restructuring of the database and, as a result, we are unable to produce the renewal forms. It is expected that renewal forms will be in the mail by the beginning of August 2002.

The membership year is from April 1 to March 31 . Members from the Atlantic provinces (Newfoundland \& Labrador, Nova Scotia, Prince Edward Island and New Brunswick) and those from Quebec renew their membership with the CTS for both the CTS and either the Atlantic Thoracic Society or the Quebec Thoracic Society. Joint membership with the CTS and these two provincial bodies is automatic.

Please note that the membership fee for the CTS has increased to $\$ 100 /$ annum effective in the $2002-2003$ year.

\section{CTS 2002 SCIENTIFIC PROGRAM Sunday, November 3, 2002 San Diego, California}

Watch for the Advance Program or visit www.chestnet.org to register!

The CTS is entering its second year of educational affiliation with the American College of Chest Physicians (ACCP). Our focus this year will be to expand our audience and capture a greater number of community-based respiratory specialists involved in clinical practice. Importantly, we are able to offer attendees Royal College of Physicians and Surgeons MAINCERT credits, now a significant issue for ongoing specialty accreditation.

The CTS wishes to contribute to the respirologist involved in clinical practice by providing:

- an excellent educational program of practical importance for the clinician;

- highlights of the best of clinical respiratory medicine in Canada today; and

- speakers from the United States and across Canada who will address topics such as asthma, infections in patients with lung disease, chronic obstructive lung disease, sleep-disordered breathing, pulmonary vascular disease, ventilator management and cystic fibrosis.

PROGRAM

What's New in Respiratory Medicine Session Chair Dr D Marciniuk

Cystic Fibrosis - Bench to Bedside

Dr B Rubin

Management of Pulmonary Hypertension Dr D McCormack

Lung Transplantation - What's Hot and What's Not

Dr R Levy

Pro-Con Debate: Advances in COPD Management Session Chair Dr P Hernandez

Clinical Implications of Pulmonary Rehabilitation Dr R Goldstein

Pro/Con Debate: Inhaled Corticosteroids Should Be Prescribed To All COPD Patients
Referee
Pro
Dr P Hernandez
Con
Dr R Hodder
Dr J Bourbeau 


$\begin{array}{ll}\text { Pro/Con Debate: Written Action Plans Improve Asthma } \\ \text { Management } & \\ \text { Referee } & \text { Dr D Cockcroft } \\ \text { Pro } & \text { Dr B Cowie } \\ \text { Con } & \text { Dr T Bai }\end{array}$

Pro/Con Debate: The New Fluoroquinolines Should Be 1st-line Therapy For Respiratory Infections in all COPD Patients
Referee
Pro
Dr G Ford
Con
Dr R Grossman
Dr R McFadden

Update on Clinical Respiratory Medicine

Session Chair

Dr H Ramsdale

Effective Ventilator Strategies

Dr Tom Stewart

Sleep Apnea and the Nasal Airway Dr M Fitzpatrick

This program has been supported thus far through unrestricted educational grants from Aventis Canada, AstraZeneca Canada, Boehringer Ingelheim Canada, Bristol-Myers Squibb Canada and Janssen Ortho Inc.

\section{CHRISTIE MEMORIAL LECTURE AND BANQUET}

Christie Memorial Lecture -

Monday November 4, 2002

Christie Memorial Annual Banquet -

Saturday, November 2, 2002, 18:30 to 20:00

The Christie Memorial Lecture honours the memory of one of the major leaders in respiratory research and education in Canada - Dr Ronald Christie. Dr Christie was an inspiration in the development of modern respiratory medicine in Canada and was renowned as dean and head of the Department of Medicine at McGill University, Montreal, Quebec, as well as being a major supporter of the CTS. The lecturer is chosen on the basis of his or her major contributions to the development of research, education or clinical excellence in the field of Canadian respiratory medicine. New this year, the Christie Memorial Lecture will be an hour-long lecture featured as part of the ACCP Honor and Memorial Lecture Series.

The 2002 Christie Memorial Lecturer is Dr Eliot A Phillipson FRCP. Dr Phillipson is chair of and professor in the Department of Medicine at the University of Toronto, Ontario. He is currently president of the Canadian Institute of Academic Medicine. His research interests include regulation of respiration in wakefulness and sleep; disorders of breathing during sleep; and the pathophysiological and clinical consequences of sleep apnea. Dr Phillipson's past honours include Gold Medal, Faculty of Medicine, University of Alberta; Cecile Lehman Mayer Research Award, American College of Chest Physicians (1973); Simon Rodbard Memorial Lecturer, American College of Chest Physicians (1990); and the Sandoz Canada Award, Clinical Research Society of Toronto (1992).

The Christie Memorial Annual Banquet will honour Dr Eliot A Phillipson. This event is an opportune time to socialize with your Canadian colleagues. The banquet and lecture are complimentary to all members of the CTS active at the time of the lecture. All other individuals interested in attending the Annual Banquet and Christie Memorial Lecture can either become a member of the Society or purchase a ticket to the event. For membership and/or ticket information, please contact Ms Valoree McKay at the Canadian Lung Association by calling 613. 569-6411 ext 222, or via email at vmckay@lung.ca

The Christie Memorial Annual Banquet is supported by a grant from Merck Frosst Canada Ltd at the platinum level and Bristol-Myers Squibb Canada at the silver level.

\section{MOVING?}

Are you moving? Please us know. Contact: Canadian Thoracic Society, 3 Raymond Street, Suite 300, Ottawa, Ontario K1R 1A3. Telephone 613-569-6411 ext 222, fax 613-569-8860, e-mail vmckay@lung.ca

\section{COMITÉ DES ESSAIS CLINIQUES}

Le comité des essais cliniques est un comité spécial de la Société canadienne de thoracologie (SCT) de l'Association pulmonaire du Canada qui compte des représentants de la Canadian Nursing Respiratory Society, de la Société de physiothérapie cardiorespiratoire du Canada et de la Société de la thérapie respiratoire de l'Association pulmonaire du Canada. Le comité vise à favoriser la recherche nationale multicentre coopérative sur des sujets cliniques importants en médecine pulmonaire adulte. En particulier, des études plus vastes sont nécessaires pour accroître le taux d'éléments probants ou de recommandations clés dans les directives de traitement présentées par la SCT. D'autres secteurs de la médecine pulmonaire, comme la pédiatrie et la chirurgie thoracique, seront explorés lorsque le comité et que ses activités seront établis.
Le groupe d'essais cliniques accueille les lettres d'intention de la collectivité respiratoire relativement aux études thérapeutiques, diagnostiques et de cohortes. Le groupe compte des représentants de toutes les universités canadiennes, mais il accepte également les projets des médecins généralistes. Le consortium sera particulièrement heureux de recevoir des projets reliés à des troubles respiratoires moins courants et désire faciliter un processus visant à améliorer la prise en charge de ces maladies. Le mandat du groupe figure à l'adresse www.lung.ca/cts, de même que la liste des représentants actuels des établissements et de leurs substituts. De plus, un canevas permettant de soumettre des protocoles au comité se trouve dans ce site Web.

\section{COMITÉ DES MEMBRES}

Certains ont peut-être remarqué qu'ils n'ont pas encore 
reçu leur formulaire de cotisation pour 2002-2003. Nous accusons un certain retard dans la restructuration de la base de données et, par conséquent, nous sommes incapables de produire les formulaires. Nous prévoyons vous les poster au début d'août 2002.

L'année de cotisation est du $1^{\text {er }}$ avril au 31 mars. Les membres des provinces atlantiques (Terre-Neuve et Labrador, Nouvelle-Écosse, Île-du-Prince-Édouard et Nouveau-Brunswick) et du Québec versent leur cotisation à la SCT avec celle de la Société de thoracologie de l'Atlantique ou de la Société de thoracologie du Québec. L'adhésion conjointe à la SCT et à l'un de ces deux organismes provinciaux se fait automatiquement.

Nous tenons à souligner que les frais de cotisation à la SCT sont passés à 100 \$ par année depuis l'exercice 20022003.

\section{PROGRAMME SCIENTIFIQUE} DE LA SCT 2002

Le dimanche 3 novembre 2002

San Diego (Californie)

Vous recevrez le programme provisoire sous peu. Vous pouvez aussi consulter le site www.chestnet.org pour vous inscrire!

La SCT poursuit une affiliation de formation avec l'American College of Chest Physicians (ACCP) pour la deuxième année. Cette année, nous viserons à accroître notre auditoire et à attirer plus de spécialistes respiratoires communautaires en exercice clinique. Nous pouvons d'ailleurs offrir aux participants des crédits MDC du Collège royal des médecins et chirurgiens, qui prennent désormais beaucoup d'importance pour ce qui est de l'accréditation continue en spécialité.

La SCT désire fournir aux pneumologues qui font de l'exercice clinique :

- un excellent programme de formation d'importance pratique pour le clinicien;

- les faits saillants de la meilleure médecine respiratoire clinique actuelle au Canada;

- des conférenciers des États-Unis et du Canada qui traiteront de sujets comme l'asthme, les infections chez les patients atteints de maladie pulmonaire, la bronchopneumopathie chronique obstructive (BPCO), les troubles respiratoires du sommeil, la maladie pulmonaire du sommeil, la prise en charge respiratoire et la fibrose kystique.

\section{PROGRAMME}

Quoi de neuf en médecine respiratoire

Président de la séance Docteur D Marciniuk

La fibrose kystique : Du laboratoire au chevet du patient

Docteur B Rubin
La prise en charge de l'hypertension pulmonaire
Docteur D McCormack

La greffe pulmonaire : Ce qui a du succès et les nouveautés

$$
\text { Docteur R Levy }
$$

Débat : Les progrès dans la prise en charge de la $\mathrm{BPCO}$

Président de la séance

Docteur P Hernandez

Les répercussions cliniques de la

réadaptation pulmonaire

Docteur R Golsdstein

Débat : Les corticoïdes par aérosol devrait être prescrits à tous les patients atteints de BPCO
Arbitre
Pour
Contre
Docteur P Hernandez
Docteur R Hodder
Docteur J Bourbeau

Débat : Des plans d'action écrits améliorent la prise en charge de l'asthme
Arbitre
Pour
Contre
Docteur D Cockcroft
Docteur B Cowie
Docteur T Bai

Débat : Les nouvelles fluoroquinolines devraient être le traitement de choix des infections respiratoires chez tous les patients atteints de $\mathrm{BCPO}$
Arbitre
Pour
Contre
Docteur G Ford
Docteur R Grossman
Docteur R McFadden

Mise à jour en médecine respiratoire clinique

Président de la séance

Docteur H Ramsdale

Des stratégies de ventilation efficaces

Docteur Tom Stewart
L'apnée du sommeil et les voies nasales
Docteur M Fitzpatrick

Jusqu'à présent, ce programme est soutenu par des subventions d'éducation sans restriction d'Aventis Canada, d'AstraZeneca Canada, de Boehringer Ingelheim Canada, de Bristol-Myers Squibb Canada et de Janssen Ortho Inc.

\section{BANQUET ET CONFÉRENCE \\ COMMÉMORATIVE CHRISTIE 2002 \\ Conférence commémorative Christie \\ Le lundi 4 novembre 2002 \\ Banquet commémoratif annuel Christie}

Le samedi 2 novembre 2002, 18 h 30 à 20 h

La conférence commémorative Christie honore la mémoire de l'un des principaux chefs de file de la recherche et de la formation en pneumologie au Canada, le docteur Ronald Christie. Le docteur Christie a inspiré le développement de la médecine respiratoire moderne au Canada, était réputé à titre de doyen et de chef du département de médecine de l'université McGill, à Montréal, au Québec et était un fervent partisan de la SCT. Le conférencier est choisi selon ses contributions au développement de la recherche ou de la 
formation ou selon son excellence clinique en médecine respiratoire au Canada. Pour la première fois cette année, la conférence commémorative Christie, d'une durée d'une heure, sera intégrée à la série de conférences commémoratives et d'hommages de l'ACCP.

Le conférencier commémoratif Christie 2002 est le docteur Eliot A Phillipson, FRCP. Le docteur Phillipson est chef et professeur au département de médecine de l'université de Toronto, en Ontario. Il préside l'Institut canadien de médecine académique. Ses intérêts en recherche incluent la stabilisation de la respiration pendant l'éveil et le sommeil, les troubles respiratoires du sommeil et la physiopathologie et les conséquences cliniques de l'apnée du sommeil. Le docteur Phillipson a déjà reçu la médaille d'or de la faculté de médecine de l'université de l'Alberta, le prix de recherche clinique Cecile Lehman Mayer de l'American College of Chest Physicians (1973), le prix du conférencier commémoratif Simon Rodbard de l'American College of Chest Physicians (1990) et le prix Sandoz Canada de la Clinical Research Society of Toronto (1992).

Le banquet annuel commémoratif Christie rendra hommage au docteur Eliot A Phillipson. Ce banquet est un moment idéal de socialiser avec vos collègues canadiens. Le banquet et la conférence sont gratuits pour tous les membres actifs de la SCT au moment de la conférence. Tous les autres particuliers intéressés à participer au banquet annuel et à la conférence commémorative Christie peuvent soit devenir membres de la Société, soit acheter un billet pour l'événement. Pour devenir membre ou obtenir des renseignements au sujet des billets, prenez contact avec madame Valoree McKay de l'Association pulmonaire du Canada, par téléphone au (613) 569-6411, poste 222, ou par courriel à vmckay@lung.ca.

Le banquet commémoratif annuel Christie est soutenu par une subvention platine de Merck Frosst Canada Ltée et par une subvention argent de Bristol Myers Squibb Canada.

\section{VOUS DÉMÉNAGEZ ?}

Vous déménagez ? Informez-nous-en. Communiquez avec la Société canadienne de thoracologie, 3, rue Raymond, bureau 300, Ottawa (Ontario) K1R 1A3. Téléphone : (613) 569-6411, poste 222, télécopieur : (613) 569-8860, courriel : vmckay@lung.ca 


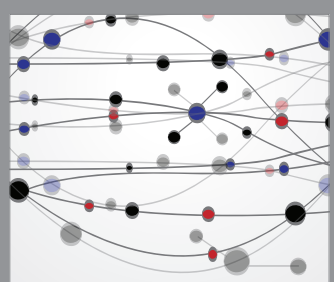

The Scientific World Journal
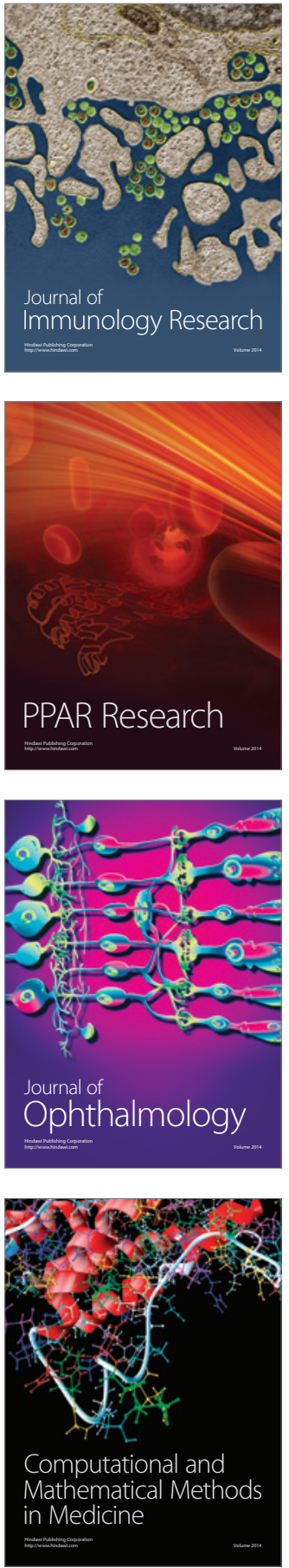

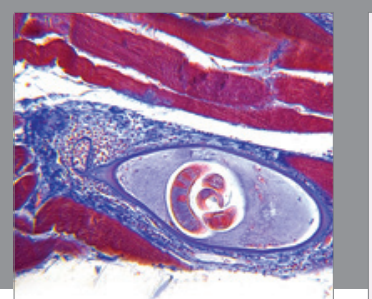

Gastroenterology Research and Practice

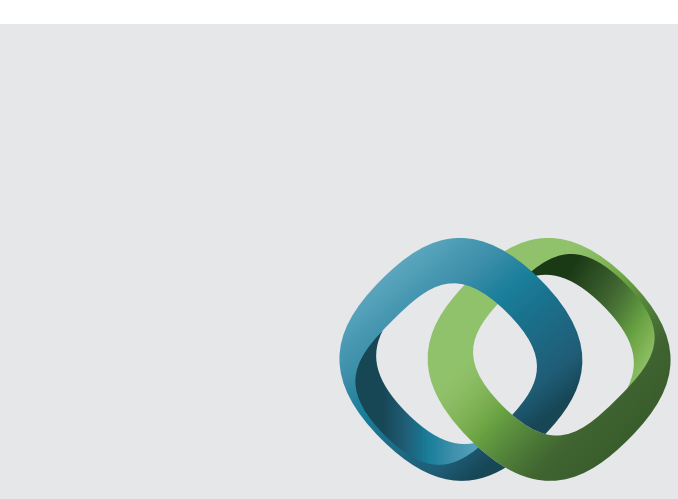

\section{Hindawi}

Submit your manuscripts at

http://www.hindawi.com
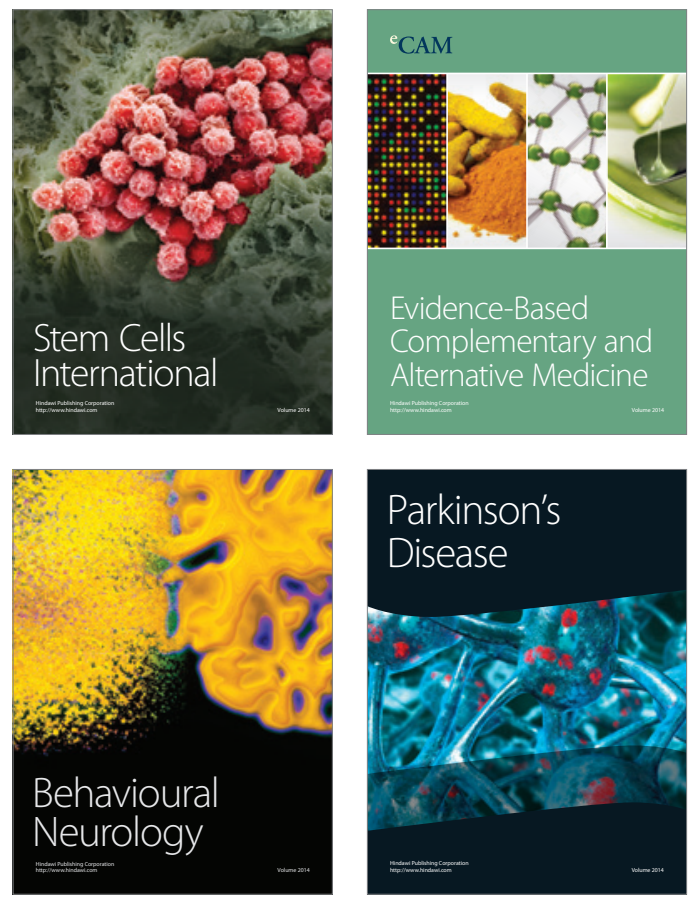
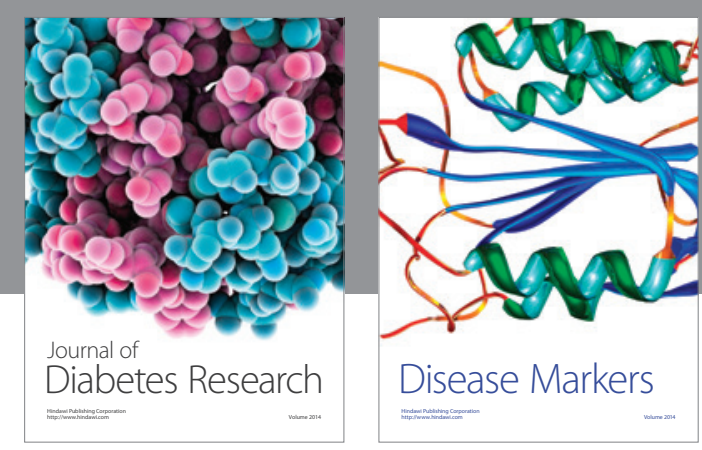

Disease Markers
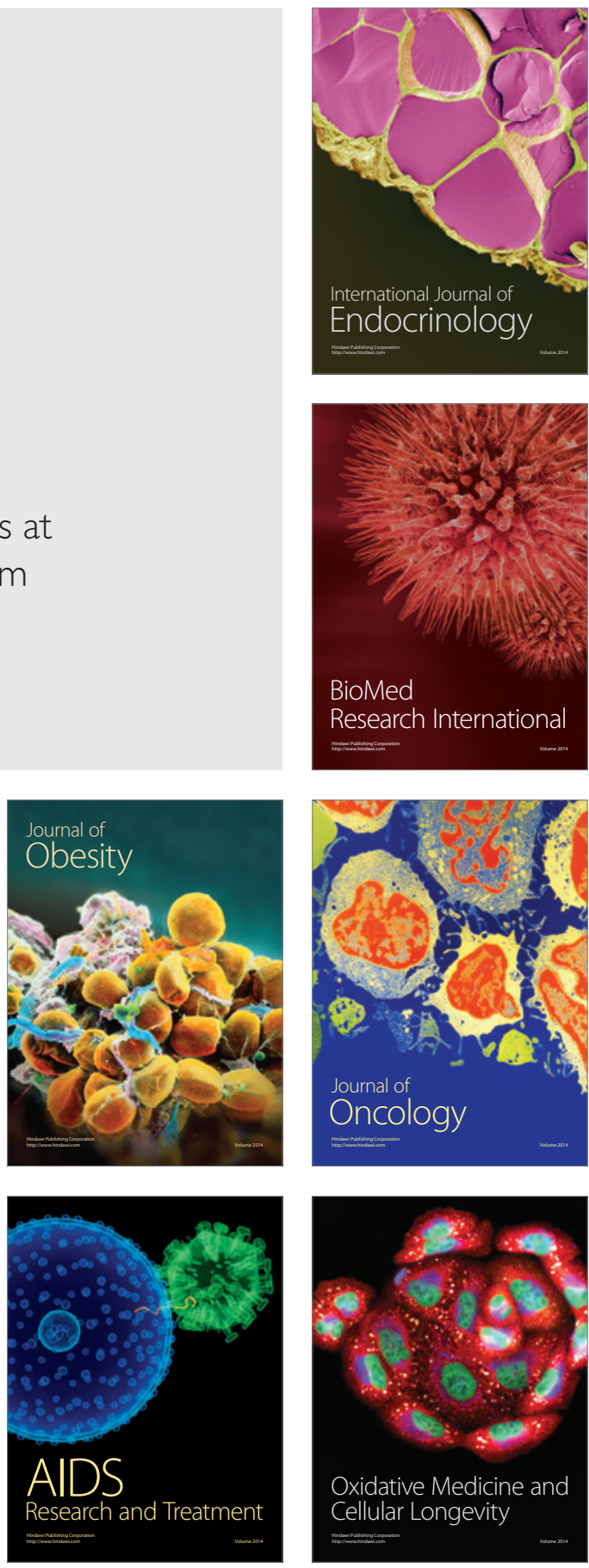